\title{
Simultaneous Measurement of Burning Velocity and Quenching Distance in Propane/Air Laminar Flames
}

\author{
Augustine Kwaku BoAKYe*, Toshihiko OHSAWA*, Tadao OzaKI, \\ Tsuyoshi Tone* and Atsuko TAKAHARA*
}

We describe a simple method for the simultaneous determination of burning velocity and quenching distance in laminar flames from measurement of flame propagation speeds. The method is applied to determine these two parameters in propane/air laminar flames at different equivalence ratios. The maximum burning velocity obtained using our method is higher than any previously reported value. The theory behind the new approach is also described.

Key Words : burning velocity, quenching distance, laminar flame, combustion diagnosis

\section{Introduction}

Burning velocity of premixed laminar flame is defined as the velocity, relative to the unburnt gases with which a plane, one dimensional flame front travels along the normal to the surface. Though its theory is simple, its absolute practical measurement is very difficult. Thus several conflicting values of burning velocity has appeared in the literature.

Various methods has been used for the measurement of burning velocity. These methods can be classified into two groups: those involving propagating flames, and those involving stationery flames. For stationery flames, the burner method has been used extensively, while for propagating flames, the constant volume bomb is the best known. A good review of the various methods is given in reference 1 . One other method that is used for the measurement of burning velocity in propagating flames is the tube method. Some authors have rigourously analysed the behaviour of this kind of flame ${ }^{1)-4}$. However little practical work has been done on it. The method is described as unreliable ${ }^{5)}$, and can never be used to obtain absolute burning velocities. The difficulties of measuring burning velocity using this approach have been described elsewhere ${ }^{6,7)}$. In this study, we propose a simple method for simultaneous measurement of burning velocity and quenching distance, using 8 different vinyl tubes. A simple theoretical model is also described. Quantitative comparison is made between the values obtained using this new approach with those previously reported in the literature.

\footnotetext{
* Faculty of Engineering, Tokyo Nohkoh University, 2-24-16, Nakamachi, Koganei

(Received July 13, 1994)

(Revised January 17, 1995)
}

\section{Theory}

Using the standard approach, burning velocity can be expressed as,

$$
\frac{\partial \rho_{u} V_{u}}{\partial t}=-\rho_{u} A S_{u}
$$

where $V_{u}$ is the unburnt gas volume, $t$ is the time interval between flame propagation between the two fixed points, $\rho_{u}$ is the unburnt gas density, $A$ is the flame surface area, and $S_{u}$ is the burning velocity.

Assuming the mixture of air-fuel is practically homogeneous, then the unburnt gas density, $\rho_{u}$, becomes a constant, hence equation 1 reduces to

$$
\frac{\partial V_{u}}{\partial t}=-A S_{u}
$$

If " $a$ " is the cross sectional area of the flame and $S_{s}$ is the flame propagation speed ahead of the flame front, it can be shown that,

$$
\frac{\partial V_{u}}{\partial X}=-a
$$

and

$$
\frac{\partial X}{\partial t}=S_{s}
$$

Where $X$ is in the direction of flame propagation to the closed end of the tube. Hence, from equations 1-4, we obtain

$$
S_{u}=\frac{a}{A} S_{s}
$$

Making the assumption that, the flame front will have a semi spherical shape throughout its propagation between the two fixed points, and making $L$ the quenching distance, from Fig. 1, it can be shown that;

$$
S_{u}=\frac{a(M)-a(L)}{A(M)-A(L)} S_{s}
$$

where $a(M)$ is the total cross sectional area of the flame without quenching, $a(L)$ is the cross sectional area of the quenched portion of the flame, $A(M)$ is the 


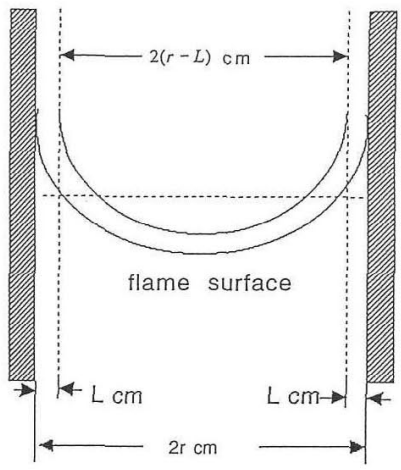

Fig. 1 Ideal model of the flame propagating in the tubes

flame area without quenching, and $A(L)$ is the estimated flame area of the quenched portion of the flame.

$$
\begin{aligned}
& A(M)=2 \pi r^{2} \\
& A(L)=2 \pi r \sqrt{2 r L-L^{2}} \\
& a(M)-a(L)=\pi(r-L)^{2}
\end{aligned}
$$

From equations $6-9$, we obtain

$$
S_{u}=\frac{(r-L)^{2}}{2 r^{2}-2 r \sqrt{\left(2 r L-L^{2}\right)}} S_{s}
$$

where $r$ is the tube radius, and $L$ is the quenching distance. Values of $S_{s}$ were obtained by the ion probes in the respective tubes. $S_{u}$ and $L$ were then inferred from a polynomial fitting of order 2, to the plot of flame propagation speed, $S_{s}$, against radius, $r$.

\section{Experimental Apparatus}

The inside diameters of the tube ranged from 0.3 $\mathrm{cm}$ to $2.5 \mathrm{~cm}$. Two identical stainless steel pins were used as electrodes. They were arranged to form the spark gaps at the center of the tubes. Two similar pairs of pins were fixed $30 \mathrm{~cm}$ from the spark electrodes, and from each other, to form the start and stop ion probes. A $0.1 \mathrm{~mm} \mathrm{Pt-Pt/Rh} 10 \%$ thermocouple was used to monitor wall temperatures of the respective tubes. It was observed that, wall temperatures did not vary significantly from room temperature, even after flame propagation. A standard piezzo electric ignition system was used for producing the spark. It did not introduce any detectable noise in the ion probes circuit. The time when the flame cuts the ion probes was measured to $\pm 0.1 \mathrm{msec}$.

A schematic representation of the experimental set-up is given in Fig. 2. The mixture of propane and air were introduced into the tubes by using a specially designed, high precision, constant pressure, gas mixer. The precision of flow regulation of this mixer is better than $0.1 \%$. Air was pumped by an air compressor, which was controlled by a relay switch, to pump air, within 5 minutes interval, into the surge tank. The air was dried and filtered, before being introduced into the surge tank. Propane $(99.9 \%$

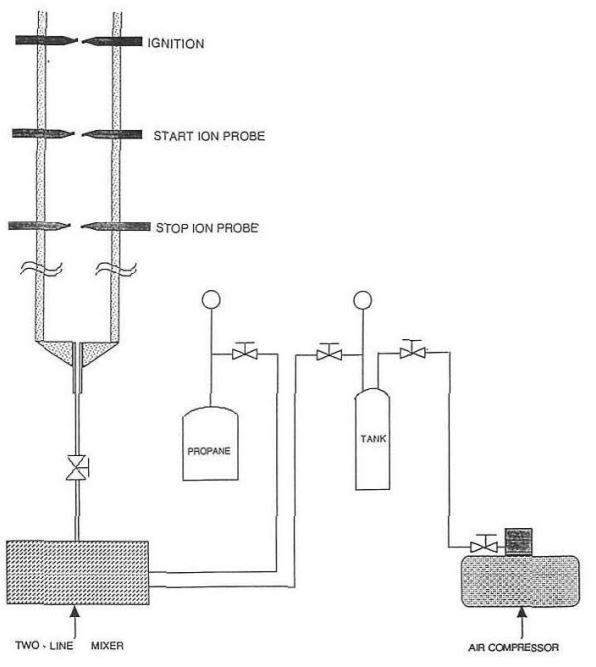

Fig. 2 Schematics of the experimental apparatus.

laboratory grade) was introduced separately into the precision mixer.

The data acquisition set-up used for our experiments consists of a pulse circuit and a universal counter to read the time difference between the points where the flame cuts the ion probes. An oscilloscope was used to monitor all inputs.

To make a measurement, the opened end of the tube is closed tightly with vinyl tape. It is then filled with the homogeneous mixture of propane and air. A waiting time of about 5 minutes was allowed to ensure that the mixture is quiescent. Measurements were then taken, by varying equivalence ratios over an extensive range. For each equivalence ratio, five runs were made to check for reproducibility.

\section{Experimental Results and Discussion}

To check for spherical symmetry of the flame fronts, initial spatial velocity distribution measurements were taken in all the tubes. Photographs were also taken to get an actual view of the flames. An example of flame photograph is shown in Photo. 1. From these two results, we concluded that, the flamefronts were nearly spherically symmetrical, except for flames in the large tubes. The reason for the latter is that, if tube diameters are large, vibrations set in, which either inhibit or accelerate the flame considerably:

Table 1 shows data for 5 identical runs in the 8 different size tubes. It can be seen that, apart from the $2.5 \mathrm{~cm}$ tube, the reproducibilities of the propagation speed measurements were very good. From these values, it can be concluded that tube radii between 0.5 and 1.5 give the most reproducible propagation speed values. 


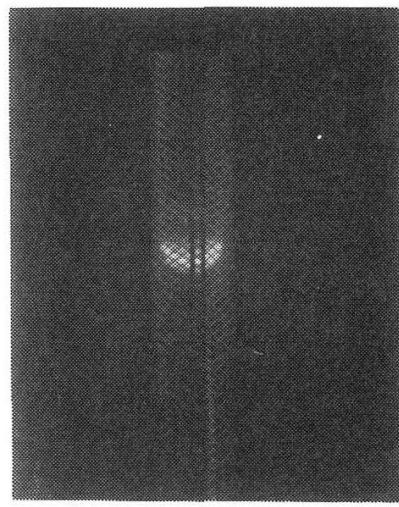

Photo.1 An example of propagating flame shape for $r=$ $0.95, \Phi=0.89$

Table 1 An example of reproducibility of propagation speed measurement for different tube radius, $r$ (cm) and for the equivalence ratio, $\Phi=1.31$

\begin{tabular}{|c|c|c|c|c|c|c|c|}
\hline \multirow[t]{3}{*}{$r$} & \multicolumn{5}{|c|}{$S_{s}$} & \multirow[t]{2}{*}{ Mean } & \multirow[t]{2}{*}{$\sigma$} \\
\hline & & & Trial & & & & \\
\hline & 1 & 2 & 3 & 4 & 5 & & \\
\hline 0.30 & 51.30 & 51.39 & 51.20 & 51.40 & 51.30 & 51.30 & 0.10 \\
\hline 0.50 & 55.00 & 55.00 & 55.30 & 55.20 & 55.40 & 55.20 & 0.20 \\
\hline 0.75 & 61.20 & 61.20 & 61.60 & 61.70 & 61.00 & 61.40 & 0.30 \\
\hline 0.95 & 58.00 & 58.00 & 60.90 & 58.80 & 61.00 & 59.70 & 1.20 \\
\hline 1.25 & 60.20 & 61.30 & 61.10 & 61.20 & 60.80 & 60.40 & 0.40 \\
\hline 1.60 & 63.70 & 63.70 & 63.80 & 62.40 & 64.10 & 63.40 & 0.70 \\
\hline 1.90 & 68.50 & 67.40 & 68.60 & 69.40 & 68.20 & 68.40 & 0.70 \\
\hline 2.50 & 60.40 & 74.40 & 74.60 & 75.30 & 74.60 & 73.70 & 2.40 \\
\hline
\end{tabular}

Fig. 3 shows typical plots of propagation speed against the radius of the tubes. A summary of derived burning velocity values are given in Table 2 . values of laminar burning velocity of propane/air laminar flame obtained by various authors, using various different methods are given in references 5,9 , and 10. Our maximum burning velocity of propane/ air laminar flame of $50.1 \mathrm{~cm} / \mathrm{s}$ is higher than any previously reported value. We are not sure of the main reason for this high value since the premixed gases were highly homogeneous and should normaly result in lower values of the burning velocity. Further experiments may be needed to clarify this. However, we are very sure that, this high value was not caused by turbulence, since the reproducibilities of the various measurements were generally good. The values of quenching distances, given in Table 2 compares favorably well with those given in reference 8 , again proving the reliability of our method.

\section{Conclusion}

We have described a very simple method for obtaining burning velocity and quenching distance simultaneously, in propane/air laminar flames. The maxi-

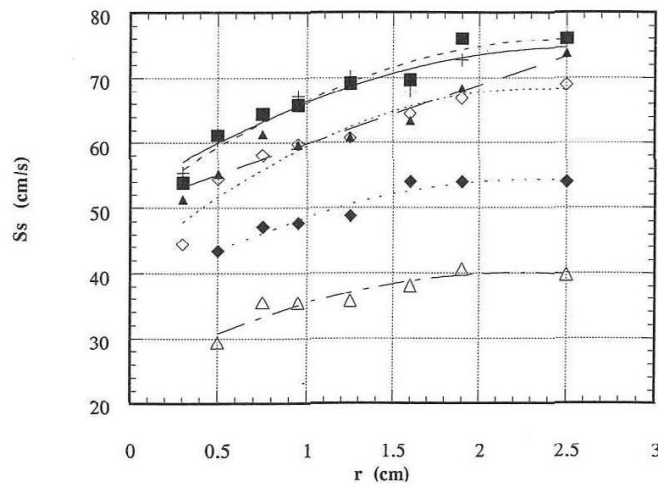

Fig. 3 Plot of flame propagating speeds against radii of the respective tubes for the equivalence ratios $1.31(\mathbf{\Delta}), 1.20(+), 1.10(\mathbf{⿴ 囗 十}), 0.99(\diamond), 0.89(\diamond)$, $0.79(\triangle)$

Table 2 Summary of derived burning velocities, $S_{u}(\mathrm{~cm} /$ s) and quenching distance, $L(\mathrm{~cm})$ values. The dispersion $S^{2}$ is also given in the table

\begin{tabular}{ccccc}
\hline \hline$\Phi$ & $S_{u}$ & $L$ & $S^{2}$ & $S^{2} / 2 S_{u}$ \\
\hline 1.31 & 41.70 & 0.06 & 3.60 & 0.04 \\
1.20 & 47.50 & 0.09 & 0.30 & 0.00 \\
1.10 & 50.10 & 0.11 & 2.50 & 0.03 \\
0.99 & 44.60 & 0.13 & 4.90 & 0.06 \\
0.89 & 36.80 & 0.15 & 0.30 & 0.00 \\
0.79 & 26.00 & 0.10 & 1.60 & 0.03 \\
\hline
\end{tabular}

mum burning velocity obtained was higher than any previously reported value. Reasonable values for quenching distances were also obtained. Within the limits of experimental errors, we believe our method is very reliable. We plan to extend our method to obtain the burning velocities of other hydrocarbons in the near future.

\section{References}

1) G.E. Andrew and D. Bradley: Combustion and Flames 18, 133/153 (1971)

2) Tony Maxworthy, The Physics of Fluids 5 (1962)

3) M. S. Uberoi: Physics of Fluids 2, 72 (1959)

4) M. S. Uberoi: Physics of Fluids, 6, 1104 (1963)

5) A. G. Gaydon and H. G. Wolfgard: Flames, Chapman and Hall Ltd 3 rd ed. (1970)

6) M. Gerstein, O. Levine and E. Wong: The Determination of Fundamental Burning Velocities of Hydrocarbons by a revised Tube Method, Journal of American Chemical soceity, 73, p. 418 (1951)

7) H.F. Coward and F. J. Hartwell: Journal of the Chemical Soceity, 1996/2676 (1932)

8) R. M. Fristrom and A.A. Westenberg: Flame struc ture, Mcgraw Hill, Inc. (U. S. A) (1951)

9) Y. Hamamoto, M. Izumi, E. Tomita, O. Miyamoto: JSME International Journal, Vol. 34, No. 2, (1991)

10) G. A. Lavoie: SAE Trans. Vol.87, Sect. 1, p. 1015, (1978) 